\title{
Limitações na Previsão de Instabilidades de Produtos Farmacêuticos Induzidos Por Radiação Durante Voos Espaciais de Longa Duração
}

\section{Limitations in Predicting Radiation-Induced Pharmaceutical Products Instabilities During Long-Term Space Flights}

\author{
Maria Nathalya Costa Souza*a; Irineu Ferreira da Silva Netoa; Isadora Ellen Feitoza Ricardinoa; \\ Ana Emília Formiga Marques ${ }^{\mathrm{a}}$
}

${ }^{a}$ Faculdade de Medicina Estácio de Juazeiro do Norte. CE, Brasil.

*E-mail: nathalya535@hotmail.com

\begin{abstract}
Resumo
Com as evoluções nos voos espaciais de humanos fora da Terra, a National Aeronautics and Space Administration (NASA) e seus parceiros internacionais encontram obstáculos relacionados à garantia da segurança dos astronautas, destacando-se a capacidade de fornecer um meio seguro e medicamentos eficazes, que possam de maneira suficiente gerenciar tanto condições médicas planejadas, quanto as imprevistas durante uma viagem espacial. Objetivou-se apresentar o entendimento atual sobre a estabilidade farmacêutica no ambiente de radiação espacial. Foi realizado um levantamento nas bases de dados científicos Nacional Center for Biotechnology Information, PubMed, SciELO e Science Direct, utilizando artigos datados entre os anos de 2016 a 2019, por meio dos descritores: Cosmic Radiation, Space Flight, Drug Stability, United States National Aeronautics e Space Administration. Foram incluídas publicações que envolvessem pesquisas na área de instabilidade de medicamentos, dentro do período delimitado, que apresentassem no idioma inglês, e que inclú́ssem pelo menos um dos descritores citados. Os produtos farmacêuticos podem se tornar instáveis através das alterações de qualquer uma das suas propriedades físicas ou químicas. Os estudos sobre voos espaciais são limitados, por isso, os desafios na tradução de evidências analógicas terrestres para voos espaciais acabam por impedir a capacidade em tirar conclusões significativas sobre a estabilidade dos produtos farmacêuticos durante a exploração espacial. Assim é necessário novos esforços em pesquisas que forneçam os dados dos voos e produtos farmacêuticos a bordo das plataformas de pesquisa disponíveis a fim de garantir a integridade da saúde da tripulação.
\end{abstract}

Palavras-chave: Fármacos. Instabilidade. Radiação. Voo Espacial.

\begin{abstract}
With the evolution in space flights of humans outside the Earth, NASA and its international partners related to ensuring the safety of astronauts, highlighting the ability to provide insurance and previous medicines, that sufficiently manage to manage both the planned medical conditions, as the unforeseen during a space trip. The objective was to present the current understanding of pharmaceutical stability in the space radiation environment. A survey was carried out in the scientific databases National Center for Biotechnology Information, PubMed, SciELO and Science Direct, using articles dated between 2016 and 2019, using the descriptors: Cosmic Radiation, Space Flight, Drug Stability, United States National Aeronautics and Space Administration. Publications involving research in the area of drug instability, within the defined period, published in the English language, and including at least one of the mentioned descriptors were published. Pharmaceutical products can become unstable by changing any of their physical or physical properties. Studies on space flights are invited, therefore, the challenges in translating analogue terrestrial evidence to space flights end up preventing the ability to take necessary to take away on the stability of pharmaceutical products during space exploration. Thus, new research efforts are needed to provide flight and pharmaceutical data on board the available research platforms to ensure the integrity of the crew's health.
\end{abstract}

Keywords: Drugs, Instability, Radiation, Space Flight.

\section{Introdução}

Com as evoluções nos voos espaciais de humanos fora da Terra, a National Aeronautics and Space Administration (NASA) e seus parceiros internacionais encontram inúmeros obstáculos relacionados à garantia da segurança dos astronautas. Dentre eles pode-se citar a capacidade de fornecer um meio seguro e medicamentos eficazes, que possam de maneira suficiente gerenciar tanto condições médicas planejadas, quanto as imprevistas durante uma viagem espacial. A possiblidade de fornecer um medicamento de forma segura e eficaz para tripulações depende de múltiplos fatores, como: a estabilidade de qualquer fármaco no período a duração de uma missão, a eficácia deste no ambiente espacial único, e o fornecimento de medicamentos que sejam adequados e suficientes para enfrentar os desafios fisiológicos e psicológicos que os astronautas podem enfrentar (NORBURY et al., 2016).

A United States Pharmacopeial (USP), fornece orientações de linhas para o conteúdo aceitável da MP (matéria prima) em medicamentos aprovados pelo Food and Drug Administration (FDA) geralmente dentro de $10 \%$ do conteúdo especificado no rótulo, embora isso pode variar consideravelmente por tipo de medicamento ou MP (MORETON, 2016). Alterações moleculares podem afetar a saturação do composto na presença ou ausência de grupos reativos, como álcoois, ácidos, cetonas, mesmo que em pequenas diferenças na estrutura essas podem afetar a rádio-sensibilidade (WOTRING, 2016). 
Existe uma escassez de evidências sobre produtos farmacêuticos e sua estabilidade no ambiente espacial, principalmente porque esse problema historicamente não era uma preocupação importante no voo espacial humano. Voos de curta duração diminuíram a necessidade de prateleira de medicamentos com vida prolongada, a seleção de membros saudáveis da tripulação minimizou a necessidade de fornecimento contínuo de medicamentos para doenças crônicas. É evidente que os cuidados com a manutenção da saúde da tripulação, regras rigorosas, as atividades perigosas durante o voo espacial, como atividade extra veicular, vêm evitando amplamente a necessidade de fornecimento de medicamentos de emergência. Mesmo agora, com missões para Estação Espacial Internacional com duração de 6 meses ou mais, equipes puderam confiar na disponibilidade de medicamentos através do descarte de medicamentos vencidos e reabastecimento frequente que possibilitam enfrentar questões de degradação, o armazenamento e o impacto do ambiente espacial (MEHTA; BHAYANI, 2017).

Diante dos dados expostos, o presente estudo tem como objetivo apresentar o entendimento atual de estabilidade farmacêutica no ambiente de radiação espacial. No intuito de identificar oportunidades de alto rendimento para pesquisas futuras que possam definir e mitigar melhor o risco de radiação espacial para formulário na exploração do voo espacial de longa duração.

\section{Desenvolvimento}

\subsection{Métodologia}

O presente estudo trata-se de uma revisão de literatura, com uma abordagem descritiva, partindo do princípio da instabilidade de medicamentos perante a radiação espacial. Foi realizado um levantamento nas bases de dados científicos Nacional Center for Biotechnology Information, PubMed (National Library of Medicine), SciELO (Scientific Electronic Library Online) e Science Direct, selecionando os materiais especializados na investigação da instabilidade de medicamentos no espaço. Foram utilizados para pesquisa os descritores: "Cosmic Radiation”, "Space Flight", "Drug Stability" e "United States National Aeronautics and Space Administration". Buscou-se evidências da instabilidade de medicamentos frente a radiação encontrada no espaço, apontando os riscos que essa radiação pode trazer aos medicamentos, e as possíveis soluções encontradas na literatura que poderiam amenizar esses efeitos. Para isso, realizou-se uma seleção dos estudos científicos publicados no idioma inglês.

Foram utilizados artigos datados entre os anos de 2016 a 2019, tendo como critério de seletividade publicações que envolvessem pesquisas na área de instabilidade de medicamentos, dentro do período delimitado, que apresentassem no idioma inglês, e que incluíssem pelo menos um dos descritores citados. Em contrapartida, publicações fora do período delimitado, que fossem em outros idiomas, incompletos ou que não abordavam sobre o tema foram excluídos. Incialmente foram encontrados 48 estudos, e, após aplicação dos critérios de inclusão e exclusão pelos autores, foram selecionados ao todo 12 estudos que cumpriam os requisitos para utilização após análise criteriosa.

\subsection{Influência da radiação sobre os fármacos}

Os efeitos da radiação se dão pela transferência de energia de uma partícula carregada para o meio pelo qual viaja. Enquanto a quantidade de energia que pode ser transferida se dá em função da cinética da partícula, energia, carga e massa. Os efeitos da ionização indireta, neste caso a radiação (por exemplo, gama e raio-x), são desprezíveis em comparação com os efeitos ocasionados pelo risco ionizante direto de partículas carregadas (ANTONSEN, 2017).

Os produtos farmacêuticos podem se tornar instáveis através das alterações de qualquer uma das suas propriedades físicas ou químicas. As modificações físicas incluem: alterações de aparência ou consistência, já as químicas incluem perda de potência, alteração dos excipientes, interações excipiente-ingrediente ativos e degradação tóxica. A fim de determinar se um medicamento náutico é inalterado pela exposição ao ambiente de radiação, este deve demonstrar, após a exposição, não sofrer alterações significativas em suas matérias primas (MP) e, ao mesmo tempo, não apresentar um desenvolvimento significativo de produtos de degradação que são tóxicos a si mesmos ou que podem de alguma forma alterar as propriedades do medicamento original (MEHTA; BHAYANI, 2017).

\subsection{Exemplos de fármacos instabilizados pela radiação}

Um medicamento pode ser considerado rádio sensível se a concentração da MP não atender as especificações da USP após a exposição à radiação. As alterações que as MPS sofrem podem afetar a potência, eficácia e segurança do medicamento, tornando-o menos eficaz ou potencialmente perigoso. Existem inúmeros casos documentados de produtos farmacêuticos sendo alterados pela exposição à radiação em doses de esterilização (25-50 kGy), por exemplo, a hidrometoclopramida cloreto produziu uma série de produtos de degradação após a exposição do mesmo à radiação 33 e a esterilização gama de certas substâncias beta demonstrou que os bloqueadores alteram os produtos farmacêuticos em relação a cor e a aparência, além de afetarem o ponto de fusão de doseamento de compostos moleculares semelhantes que podem ter respostas muito diferentes à irradiação (DANIELS et al., 2017).

A cefradina e cefotaxima, ambos antibióticos da classe das cefalosporinas, na sua forma sólida de estrutura molecular demonstram diferenças de rádio-sensibilidade quando expostos a doses idênticas de esterilização de radiação gama. A cefradina degrada significativamente e tem sido determinada 
como instável sob irradiação, enquanto a cefotaxima demonstra alta resistência e estabilidade (HUFF et al., 2016).

No Quadro 1 são citados alguns medicamentos utilizados em voos espaciais, relacionando-os com seu grau de resistência à radiação e os tipos de alterações que podem sofrer, essas restritas as suas matérias-primas, características físicas e formação de produtos de degradação.

Quadro 1 - Relação dos medicamentos com seu grau de resistência à radiação e tipo de alteração

\begin{tabular}{|c|c|c|c|}
\hline $\begin{array}{l}\text { Autor/ } \\
\text { Ano }\end{array}$ & Medicamento & $\begin{array}{c}\text { Grau de } \\
\text { Resistência }\end{array}$ & Tipo de Alteração \\
\hline \multirow{12}{*}{$\begin{array}{l}\text { Blue et } \\
\text { al. } 2019\end{array}$} & Ibuprofeno & Forte & $\begin{array}{l}\text { Matéria-prima } \\
\text { Características físicas } \\
\text { Forma produtos de } \\
\text { degradação }\end{array}$ \\
\hline & Epinefrina & Fraco & $\begin{array}{l}\text { Matéria-prima } \\
\text { Características físicas }\end{array}$ \\
\hline & Amoxicilina & Fraco & $\begin{array}{l}\text { Matéria-prima } \\
\text { Características físicas }\end{array}$ \\
\hline & Fluconazol & Fraco & $\begin{array}{l}\text { Matéria-prima } \\
\text { Características físicas }\end{array}$ \\
\hline & Furosemida & Fraco & $\begin{array}{l}\text { Matéria-prima } \\
\text { Características físicas }\end{array}$ \\
\hline & $\begin{array}{l}\text { Suplemento de } \\
\text { vitamina D }\end{array}$ & Forte & Matéria-prima \\
\hline & $\begin{array}{c}\text { Multivitamínico } \\
\text { uma vez ao dia } \\
\text { das mulheres }\end{array}$ & Fraco & $\begin{array}{l}\text { Matéria-prima } \\
\text { (Vitamina D) }\end{array}$ \\
\hline & Aspirina & Forte & $\begin{array}{l}\text { Matéria-prima } \\
\text { Forma produtos de } \\
\text { degradação }\end{array}$ \\
\hline & Zolpidem & Forte & $\begin{array}{l}\text { Matéria-prima } \\
\text { Forma produtos de } \\
\text { degradação }\end{array}$ \\
\hline & Sertralina & Fraco & $\begin{array}{l}\text { Matéria-prima } \\
\text { Características físicas }\end{array}$ \\
\hline & Lidocaína & Fraco & $\begin{array}{l}\text { Matéria-prima } \\
\text { Características físicas }\end{array}$ \\
\hline & $\begin{array}{l}\text { Multivitamínico } \\
\text { Centrum Silver }\end{array}$ & Forte & Matéria-prima \\
\hline
\end{tabular}

\section{Fonte: Dados da pesquisa.}

Perante a pesquisa de Blue et al. (2019), verifica-se que existem medicamentos que são mais resistentes a exposição à radiação em voos espaciais. Os mais sensíveis sofrem alterações relacionadas com maior índice de formação de produtos de degradação, alterações em suas matérias-primas e nas características físicas, enquanto que os menos sensíveis inicialmente apresentam alterações apenas na sua aparência, mas podem também, dependendo do tempo de exposição e da dose, apresentarem alterações nos parâmetros supracitados.

\subsection{Instabilidade de fármacos em voos espaciais}

Os estudos sobre voos espaciais são limitados, e os desafios na tradução de evidências analógicas terrestres para voos espaciais acabam por impedir a capacidade em tirar conclusões significativas sobre a estabilidade dos produtos farmacêuticos durante a exploração espacial. Enquanto a NASA enfrenta os desafios associados a missões que envolvem maior distância da Terra, a atual incapacidade de fornecer uma farmácia segura e eficaz para a exploração e o voo espacial é, de fato, uma grande lacuna. Por isso, ressalta-se a importância de um estudo como esse, tanto para atualizar os dados sobre a temática, quanto também para instigar o desenvolvimento de novas pesquisas. Para resolver esse problema, a NASA desenvolveu recentemente um Pharmacy Research, sendo um plano em que a estabilidade farmacêutica e o risco de radiação são destacados como incógnitas que devem ser abordadas em esforços de pesquisas anteriores às missões a Lua ou a Marte. $\mathrm{O}$ plano de pesquisa enfrenta diversos desafios, incluindo o curto prazo de congelamento do projeto e a necessidade de declarar missões de exploração de rápida aproximação, com expectativa de ocorrer na próxima década (JAWORSKE; MYERS, 2016).

\subsection{Estratégias que podem ser adotadas para reduzir a instabilidade}

A partir do uso de excipientes acredita-se ser possível alcançar uma maior estabilidade em um ambiente de radiação. Por exemplo, formulações incluindo amido, estearato, celulose e a dextrose podem ser mais estáveis. Outras opções incluem preparações com os excipientes como manitol, nicotinamida, e piridoxina, que demonstraram propriedades radioprotetoras e laços no processamento da esterilização terrestre (MEHTA; BHAYANI, 2017).

Deve-se reiterar que grande parte da literatura que apoie a inclusão ou exclusão de excipientes para propriedades protetoras ou de estabilidade são novamente baseadas em dados, amostragem de conveniência ou exposições a radiações diferentes do ambiente espacial, limitando a tradução de descobertas, particularmente, para missões de exploração de longa duração. Além disso, alterar ou adicionar excipientes mudaria a formulação do medicamento, o produto resultante seria considerado um novo medicamento e, conforme regulamentos da USP, exigiria um novo pedido de aprovação e demonstração de segurança, potência e falta de substâncias tóxicas. Em esforços colaborativos para proteger a tripulação humana de exposição à radiação, muitas discussões vêm acontecendo a respeito da inclusão de um compartimento fortemente blindado de alumínio grosso ou outro material radioprotetor ou, alternativamente, o uso de "soluções de blindagem multiuso" como barreiras compostas por água ou alimentos, em veículos de exploração (JAWORSKE; MYERS, 2016).

Os medicamentos podem ser armazenados dentro de um compartimento blindado para reduzir as exposições à radiação. Embora esses esforços inovadores mostrem eficiência, é importante lembrar que os projetos de blindagem podem ser limitados por restrições de massa e volume e a capacidade de elevação do veículo lançador. É prematuro assumir que a blindagem idealizada será implementada com sucesso nos veículos de exploração. Muitos dos designs de escudo destinam-se a implantação "just-in-time" para proteção da tripulação, em tais circunstâncias a tripulação 
precisaria recuperar os medicamentos a bordo para armazenálos protegidos, mesmo se fosse necessário a implementação de um alto grau de blindagem, tal compartimento mitigaria apenas as exposições associadas a grandes Solar Partcle Event (SPE) e exposições prolongadas ao Galactic Cosmic Radiation (GCR) continuariam a representar uma ameaça à estabilidade dos medicamentos a longo prazo (DANIELS et al., 2017).

Um plano abrangente de pesquisa a bordo pode não ser viável, dadas as restrições financeiras e de tempo, enquanto um estudo dos efeitos comparativos das exposições únicas e multienergéticas podem melhorar nossa compreensão da complexidade do espaço ambiente de radiação e seu impacto nos produtos farmacêuticos. Como discutido no presente artigo, o uso de doses e taxas de doses mais próximas podem simular o ambiente espacial, o que pode fornecer informações mais úteis ou resultados precisos do que a dependência de altas doses e exposições das mesmas. Além disso, a inclusão de materiais de proteção nas áreas terrestres de design analógico, para atenuar a dose e simular o potencial de reações de fragmentação, pode imitar melhor a composição iônica de ambiente intraveicular (CHANCELLOR et al., 2018).

\subsection{Necessidade estudos de instabilidade de fármacos em voos espaciais}

A mitigação bem-sucedida do risco de radiação depende de uma compreensão mais aprofundada dos efeitos potenciais da radiação sobre produtos farmacêuticos, informações sobre quais produtos farmacêuticos correm maior risco de danos induzidos por radiação e um conhecimento de como a infinidade de fatores relacionados ao voo espacial, por exemplo, farmacocinética e farmacodinâmica alteradas, dose de radiação, taxa de dose de radiação, embalagem, prazo de validade, entre outros, afetam os medicamentos. Um estudo cuidadoso e controlado da estabilidade farmacêutica, com controles de solo e tamanho apropriado da amostra, melhoraria a compreensão dos riscos multifatoriais para os medicamentos no espaço. Estudos adicionais baseados no solo comparando os efeitos da radiação gama, raio-x ou feixe de elétrons para prótons ou íons pesados, pode melhorar a compreensão de como traduzir melhor a literatura terrestre ao contexto da radiação espacial (BLUE et al., 2019).

A coleta sistemática de dados para caracterização de medicamentos, uso, eficácia, efeitos colaterais, farmacocinética, farmacodinâmica e estabilidade a longo prazo, têm sido uma importante prioridade. Com o empurrão para missões de exploração na Lua e Marte, essas perguntas recebem maior atenção. Um risco potencial para a estabilidade farmacêutica depende da exposição a longo prazo ao ambiente de radiação espacial. A exposição à radiação gama tem sido usada na terra para esterilização em produtos farmacêuticos selecionados, já a radiação espacial diferenciase consideravelmente devido as práticas e as diferenças no tipo de radiação, dose, taxa de dose e duração da exposição, ou seja, não está claro se a exposição a longo prazo à radiação espacial pode afetar a estabilidade, alterar ingredientes ou produzir substâncias potencialmente tóxicas, particularmente em medicamentos que sofreram degradação (JONSCHER et al., 2016).

A utilização da International Space Station - ISS como plataforma de pesquisa, com armazenamento de longa duração de medicamentos e estudos bem projetados e controlados de prateleira com exposição à vida e à radiação, poderia fornecer informações essenciais relacionadas a estabilidade de voo espacial. Contudo, esses estudos precisariam ser iniciados rapidamente, pois a ISS é destinada a maior frequência de viagens na próxima década. Com a aproximação rapidamente das datas das missões de exploração, a NASA e seus parceiros internacionais buscam um formulário farmacêutico maduro que pode ser realizado antes do congelamento do design do veículo e das missões que tendem a ocorrer (BLUE et al., 2019).

Então, a inclusão de produtos farmacêuticos, particularmente novos ou complexos medicamentosos, no formulário da ISS para futuras missões tripuladas ou não tripuladas fora da Low Eart Orbit (LEO) podem fornecer dados adicionais sobre a estabilidade dos medicamentos no ambiente espacial. No entanto, a maioria dessas missões não devolve cargas úteis à Terra, limitando as opções de análise. Logo, para alavancar as oportunidades de análise é necessário compromissos programáticos para o retorno da amostra, que por sua vez depende de um aumento da compreensão e da importância desses dados (DANIELS et al., 2017).

\section{Conclusão}

Estudos comparativos de medicamentos, incluindo e excluindo materiais de blindagem ou embalagem, podem fornecer informações sobre a contribuição relativa de tais materiais às reações de degradação farmacêutica. Uma avaliação cuidadosa e completa dos produtos farmacêuticos expostos ao ambiente espacial seria necessária dada a escassez de dados apropriados de estudos para a compreensão de exposições à radiação espacial. É fundamental abordar esses conhecimentos antes das missões para a Lua ou Marte estarem em andamento. Tendo em vista que existem avanços significativos que podem ser alcançados através de um planejamento eficaz.

Esforços em pesquisas que forneçam os dados dos voos e os produtos farmacêuticos a bordo das plataformas de pesquisa disponíveis, como o ISS, bem como estudos translacionais de efeitos comparativos de como a radiação age em análogos da terra, permitem uma melhor interpretação da compreensão histórica da radiação terrestre no contexto do voo espacial. Outra alternativa seria a utilização de técnicas de modelagem aprimoradas e robustas para simular melhor o ambiente espacial, um estudo cuidadoso das várias formulações, opções alternativas de medicamentos e embalagens além da consideração de novas técnicas, como armazenamento 
criogênico, poderiam fornecer avanços de suma importância para o desenvolvimento de uma capacidade farmacêutica para os voos espaciais de longa duração.

\section{Referências}

ANTONSEN, E. Risk of Adverse Health Outcomes and Decrements in Performance Due to In-flight Medical Conditions. Houston, 2017.

BLUE, R.S. et al. Limitations in Predicting Radiation-Induced Pharmaceutical Instability During Long-Duration Spaceflight, Houston, TX, United States, 2019.

CHANCELLOR, J.C. et al. Limitations in predicting the space radiation health risk for exploration astronauts. NPJ Microgravity, v.4, n.1, p.1-11, 2018. doi: 10.1038/s41526-018-0043-2

DANIELS, V.R et al. The Pathway to a Safe and Effective Medication Formulary for Exploration Spaceflight, Houston, 2017.

HUFF, J. et al. Evidence Report: risk of radiation carcinogenesis.
Houston, 2016.

JAWORSKE, D.A; MYERS, J.G. Pharmaceuticals exposed to the space environment: problems and prospects. Cleveland, $\mathrm{OH}$ United States, 2016.

JONSCHER, K.R. et al. Correction: spaceflight activates Lipotoxic pathways in mouse liver. PloS one, v.11, n.5, p. e0155282, 2016. doi: 10.1371/journal.pone.0155282

MEHTA, P.; BHAYANI, D. Impact of space environment on stability of medicines: challenges and prospects. J. Pharm. Biom. Anal., v.136, p.111-119, 2017. doi: 10.1016/j.jpba.2016.12.040

MORETON, R.C. United States Pharmacopeia-National Formulary. J. Excipients Food Chem., v.6, n. 3, p. 925, 2016.

NORBURY, J.W. et al. Galactic cosmic ray simulation at the NASA Space Radiation Laboratory. Life Scie. Space Res., v.8, p.38-51, 2016. doi: 10.1016/j.1ssr.2016.02.001

WOTRING, V.E. Chemical potency and degradation products of medications stored over 550 earth days at the international space station. AAPS J., v.18, n.1, p.210-216, 2016. doi: 10.1208/ s12248-015-9834-5 